\title{
Influencia de los lazos sociales en la salud de las personas
}

Influence of social ties on people's health

Mariano Granero, Camila Volij, Santiago Esteban y Sergio Terrasa ¥

\begin{abstract}
Resumen
Se denomina apoyo social (AS) a la información y/o ayuda que recibe o percibe recibir una persona, con repercusión conductual y emocional. El AS estructural se refiere a los aspectos objetivos y/o cuantitativos de la red social, mientras que el funcional hace hincapié en los efectos que generan en un sujeto la conservación de su relaciones sociales. Se denomina AS objetivo a los recursos, las provisiones y las transacciones reales a los que las personas pueden recurrir. EI AS percibido integra aspectos subjetivos de apoyo social y se centra en lo que percibe la persona en cuestión respecto de la ayuda con la que cree contar, existiendo creciente evidencia de su importancia pronóstica a través de caminos causales conductuales, psicológicos y fisiológicos.

\section{Abstract}

Social support (AS) includes information and/or help received or perceived by a person, with behavioral and emotional consequences. Structural AS refers to objective and/or quantitative aspects of the social network, while functional AS emphasizes the effects generated by the preservation of social relations.

Objective AS means resources, provisions and actual transactions to which people can turn. Perceived AS integrates subjective aspects of social support and focuses on what the person in question feels with respect to the help he or she believes they have; and there is growing evidence of its prognostic importance through behavioral psychological and physiological causal paths.
\end{abstract}

Granero $\mathrm{M}^{*}$, Volij $\mathrm{C}^{*}$, y col. Influencia de los lazos sociales en la salud de las personas. Evid Act Pract Ambul. 2018;21(1):2-3.

*Estos autores contribuyeron de igual manera a este trabajo.

Palabras clave: apoyo social, pronóstico, causas. Key words: Social support, prognosis, causes.

\section{INTRODUCCIÓN}

Se entiende por relación social a la que existe entre dos o más personas cuando cada una de ellas influye en los pensamientos, sentimientos y/o comportamientos de la/s otra/s. Sin embargo, el apoyo social (AS) es un constructo multidimensional con diferentes aspectos estructurales y funcionales, por lo que su definición ha resultado dificultosa a lo largo de los años, y no se ha alcanzado aún una conceptualización definitiva y consensuada.

El AS puede definirse como la información (verbal o no verbal) y/o ayuda que recibe (sea en forma tangible/objetiva) o bien que percibe recibir (intangible/subjetiva) una persona, y que tiene efectos conductuales y emocionales en el receptor ${ }^{1}$.

\section{PRINCIPALES CONCEPTUALIZACIONES SOBRE EL APOYO SOCIAL}

El AS puede ser conceptualizado desde dos grandes puntos de vista: el que compara los aspectos funcionales respecto de los estructurales y el que hace un paralelo entre el AS objetivable respecto del AS percibido.

\section{Apoyo social estructural vs funcional}

EI AS estructural se refiere a los aspectos objetivos y/o cuantitativos de la red social (tamaño, densidad, dispersión geográfica, etc.).

El AS funcional hace hincapié en los efectos o consecuencias que generan en un sujeto el acceso y la conservación de las relaciones sociales que tiene en su red. A su vez, este AS funcional puede ser dividido en emocional (sentimiento personal de ser amado, seguridad de poder confiar en alguien y de tener intimidad con esa persona), instrumental (posibilidad de poder disponer de ayuda directa) e informacional (provisión de consejo o guía para la resolución de problemas).

Apoyo social objetivo vs percibido

Se denomina apoyo social objetivo a los recursos, las provisiones y las transacciones reales a los que las personas pueden recurrir ${ }^{2}$.

Como lo expresa su nombre, el apoyo social percibido integra aspectos subjetivos de AS y se centra fundamentalmente en lo que percibe la persona en cuestión respecto de la ayuda con la que cree contar. Como explicaremos más adelante, existe creciente evidencia de la importancia pronóstica del AS percibido respecto de la salud de las personas.

Subconstructos del apoyo social

Más allá de estos grandes conceptos, han sido descriptos por diferentes autores ${ }^{3,4,5}$ cuatro subconstructos respecto del AS, que están muy vinculados con los que mencionamos previamente:

a) El grado de integración que tiene la persona en cuestión en las redes sociales de la vida real, conocido como aislamiento social objetivo.

b) El sentimiento angustioso que acompaña la percepción de que las necesidades sociales de uno no están siendo satisfechas por la cantidad o la calidad de las relaciones sociales disponibles, denominado aislamiento social o soledad percibida.

c) Las interacciones sociales que están destinadas a ser de apoyo, también conocidas como apoyo social recibido.

d) Las creencias y percepciones respecto de la disponibilidad de apoyo que posee el individuo o apoyo social percibido.

Dado lo descripto previamente, el primer subconstructo (aislamiento social objetivo) representa los aspectos estructurales de las relaciones sociales y los tres últimos, sus aspectos funcionales.

\section{ASPECTOS EPIDEMIOLÓGICOS DE LAS RELACIONES SO- CIALES}

Existe evidencia creciente de que, dadas las características del estilo de vida actual, la calidad y la cantidad de los lazos sociales está disminuyendo progresivamente en las sociedades industrializadas. Entre sus rasgos principales se destacan la reducción de la convivencia intergeneracional en los hogares, los matrimonios tardíos, las familias en las que tanto el padre como la madre desarrollan una carrera profesional, y la tendencia a vivir solo en pequeñas viviendas en los grandes centros urbanos ${ }^{6}$. Por ejemplo, el $80 \%$ de los menores de 18 años de edad y el $40 \%$ de los adultos mayores de 65 años declaran estar solos al menos a veces ${ }^{4,7}$, con niveles de soledad que son menores durante la adultez y mayores durante la juventud y la vejez ${ }^{4}$.

‡ Servicio de Medicina Familiar y Comunitaria del Hospital Italiano de Buenos Aires mariano.granero@hospitalitaliano.org.ar 
Este fenómeno parece ser creciente, ya que estudios recientes documentaron que en los últimos 20 años se triplicó la cantidad de estadounidenses que afirman no tener ninguna persona de confianza ${ }^{8}$; mientras que en el Reino Unido, según un reciente estudio de la Fundación de Salud Mental, el 10\% de las personas se sienten solas, un tercio de las personas afirma tener un amigo cercano o pariente al que ve muy solitario, y la mitad piensa que la gente se está volviendo más solitaria en general ${ }^{3}$.

Estos datos sugieren que, a pesar del avance de la globalización y del desarrollo de tecnologías de comunicación, la gente estaría viviendo cada vez con AS de peor calidad.

\section{LA INFLUENCIA DE LAS RELACIONES SOCIALES EN LA SALUD DE LAS PERSONAS}

Durante la década de los "setenta" el aislamiento social comenzó a ser estudiado desde la investigación epidemiológica, por su asociación con la morbilidad y la mortalidad prematura ${ }^{3,9}$.

Hoy en día se sabe que la influencia de las relaciones sociales sobre la mortalidad es comparable con la de factores de riesgo bien establecidos como el tabaquismo, el sedentarismo y la obesidad.

Por ejemplo, el metanálisis realizado por Holt-Lunstad y col. ${ }^{3}$ documentó que los individuos con relaciones sociales adecuadas, tienen una probabilidad de supervivencia $50 \%$ mayor que aquellos con relaciones sociales pobres o insuficientes. Por otro lado, también se ha estudiado la relación entre el aislamiento social y/o la soledad, con la incidencia de enfermedad coronaria y accidente cerebrovascular (ACV). Por ejemplo, el metanálisis publicado por Valtorta y col. ${ }^{10}$ identificó que las relaciones sociales pobres se asocian con un aumento del $29 \%$ en el riesgo de enfermedad coronaria y un $32 \%$ de aumento en el riesgo de ACV.

\section{POTENCIALES CAMINOS CAUSALES ENTRE LAS RELA- CIONES SOCIALES Y SUS EFECTOS SOBRE LA SALUD DE LAS PERSONAS}

Si bien y como expresamos anteriormente, la influencia de las relaciones sociales sobre la mortalidad es comparable con otros factores de riesgo bien establecidos ${ }^{3}$, sabemos mucho menos sobre sus mecanismos etiopatogénicos. Sin embargo, algunas líneas de investigación han identificado tres principales caminos causales a través de los cuales las relaciones sociales afectarían la salud: 1) conductuales, 2) psicológicos, 3) fisiológicos ${ }^{3,11}$.

Entre los caminos causales conductuales vale destacar que las personas aisladas tienden a realizar menos actividad física y a fumar más ${ }^{12}$. Entre los psicológicos, la soledad está asociada a una menor autoestima y el aislamiento social, a una disminución en la autoeficacia ${ }^{13}$. Por último, el sentirse solo o estar socialmente aislado se asocia con un funcionamiento inmune defectuoso $0^{9,14}$ y además, la depresión ${ }^{15}$, que suele estar asociada a deficiencias en el apoyo social, se asocia a su vez a estados pro-inflamatorios vinculados con diversas enfermedades cardiovasculares. Ver figura 1.

\section{CONCLUSIONES}

La evidencia que hemos resumido sugiere que la soledad y el aislamiento social pueden ser factores de riesgo importantes para desarrollar enfermedades y que abordarlos eficazmente podría ser beneficioso en términos de salud pública ${ }^{10}$, y como sostiene House ${ }^{16}$, "...las relaciones sociales, o su relativa ausencia constituyen un importante factor de riesgo para la salud, rivalizando con el efecto de factores de riesgo bien establecidos para la salud como el tabaquismo, la presión arterial, los lípidos en la sangre, la obesidad y la actividad física".

Dado que esta problemática tiene un gran impacto en los adultos mayores, la Organización Mundial de la Salud promueve desde 2002 la prevención del aislamiento social como una medida necesaria para la promoción del cuidado de la salud y de un envejecimiento activo ${ }^{10,17}$

Figura 1: conceptualización de los potenciales mecanismos causales entre las relaciones sociales y la salud de las personas.

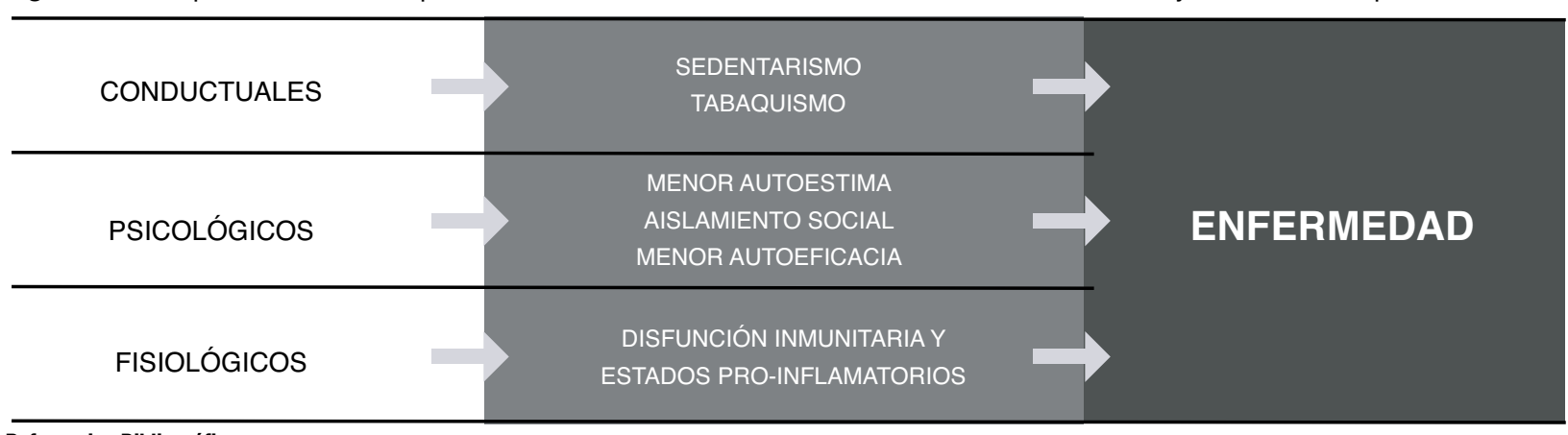

Referencias Bibliográficas

1. Aranda B y col. Conceptualización del apoyo social y las redes de apoyo social. Revista de Investigación en Psicología 16, 233 (2014).

2. Barrera, M y col. The structure of social support: a conceptual and empirical analysis. J. Community Psychol. 11, 133-143 (1983).

2. Barrera, M y col. The structure of social support: a conceptual and empirical analysis. J. Community Psychol. 11, 133-143
3. Holt-Lunstad, J y col. Social Relationships and Mortality Risk: A Meta-analytic Review. PLoS Med. 7, e1000316 (2010).

3. Holt-Lunstad, J y col. Social Relationships and Mortality Risk: A Meta-analytic Review. PLoS Med. 7, e1000316 (2010).
4. Pinquart M y col. Influences on Loneliness in Older Adults: A Meta-Analysis. Basic Appl. Soc. Psych. 23, 245-266 (2001).

4. Pinquart M y col. Influences on Loneliness in Older Adults: A Meta-Analysis. Basic Appl. Soc. Psych. 23, $245-$. Wheeler L y col. Loneliness, social interaction, and sex roles. J. Pers. Soc. Psychol. 45, 943-953 (1983).
5.

5. Wheeler $L$ y col. Loneliness, social interaction, and sex roles. J. Pers. Soc. Psychol. 45, 943-953 (1983).
6. Putnam R y col. Bowling Alone: The Collapse and Revival of American Community. (Simon and Schuster, 2001).

7. Seeman T y col. Social ties and health: The benefits of social integration. Ann. Epidemiol. 6, 442-451 (1996).

8. Caplan G y col. Support systems and mutual help: multidisciplinary explorations. (1976).

9. Grant N y col. Social isolation and stress-related cardiovascular, lipid, and cortisol responses. Ann. Behav. Med. 37, 29-37 (2009).

10. Valtorta $\mathrm{N} \mathrm{y} \mathrm{col.} \mathrm{Loneliness} \mathrm{and} \mathrm{social} \mathrm{isolation} \mathrm{as} \mathrm{risk} \mathrm{factors} \mathrm{for} \mathrm{coronary} \mathrm{heart} \mathrm{disease} \mathrm{and} \mathrm{stroke:} \mathrm{systematic} \mathrm{review} \mathrm{and} \mathrm{meta-analysis} \mathrm{of} \mathrm{longitudinal} \mathrm{observational} \mathrm{studies.}$ Heart 102, 1009-1016 (2016)

11. Hawkley L y col. Loneliness matters: a theoretical and empirical review of consequences and mechanisms. Ann. Behav. Med. 40, 218-227 (2010).

12. Shankar A y col. Loneliness, social isolation, and behavioral and biological health indicators in older adults. Health Psychol. $30,377-385$ (2011).

13. McAvay G y col. A longitudinal study of change in domain-specific self-efficacy among older adults. J. Gerontol. B Psychol. Sci. Soc. Sci. 51, P243-53 (1996).

14. Mawkley L y col. Loneliness predicts increased blood pressure: 5 -year cross-lagged analyses in middle-aged and older adults. Psychol. Aging 25, $132-141$ (2010).

14. Hawkley $\mathrm{L}$ y col. Loneliness predicts increased blood pressure: 5 -year cross-lagged analyses in middle-aged and older adults. Psychol. Aging 25 , $132-141$ (2010).
15. Slavich $\mathrm{G}$ y col. From stress to inflammation and major depressive disorder: a social signal transduction theory of depression. Psychol. Bull. 140, $774-815$ (2014).

15. Slavich $\mathrm{G}$ y col. From stress to inflammation and major depressive disorder:

17. Bronswijk J y col. Active ageing: A policy framework - WHO's ageing and life course program; 2002, by the World Health Organisation. Gerontechnology 2, (2003). 